\title{
Pattern of declining blood pressure across replicate population surveys of the WHO MONICA project, mid-1980s to mid-1990s, and the role of medication
}

Hugh Tunstall-Pedoe, John Connaghan, Mark Woodward, Hanna Tolonen, Kari Kuulasmaa

\begin{abstract}
Objective Declining mean systolic and diastolic blood pressures were observed in most populations of the World Health Organization MONICA (monitoring trends and determinants in cardiovascular disease) project from the mid-1980s to mid-1990s. We tested whether pooled results would show mean change associated with decline in high readings only, resulting from better antihypertensive medication, or with similar falls in low, middle, and high readings, implying other causes. Design Independent, random sample, cross sectional population surveys, each end of the MONICA decade. Setting 38 populations in 21 countries across four continents. Participants Design target in each survey of 200 participants in each 10 year age and sex group from age 35 to 64 Main outcome measures Changes in the population in mean systolic and diastolic blood pressure, and in low, middle, and high readings - the 20th, 50th, and 80th centiles-and the differences between these changes.

Results Individual populations differed considerably, but pooling the 38 population results gave mean changes in systolic blood pressure of $-2.2 \mathrm{~mm} \mathrm{Hg}$ in men, $-3.3 \mathrm{~mm} \mathrm{Hg}$ in women, and in diastolic blood pressure of $-1.4 \mathrm{~mm} \mathrm{Hg}$ in men and $-2.2 \mathrm{~mm} \mathrm{Hg}$ in women (overall average $-2.26 \mathrm{~mm} \mathrm{Hg}$, population median - $1.55 \mathrm{~mm} \mathrm{Hg}$ ). Antihypertensive medication, associated with high readings, rose by $0.5 \%$ to $11.4 \%$. However, average falls in low and middle blood pressure readings were so similar to those in high readings and in the mean that no effect from improving treatment of hypertension was detected. Results in contrasted subgroups were consistent. Conclusions Blood pressure fell across 38 MONICA populations at all levels of readings, with no differential fall in high readings attributable to better control of hypertension. Despite the importance of medication to individuals, in that decade other determinants of blood pressure lowering must have been more pervasive and powerful in whole populations.
\end{abstract}

\section{Introduction}

The World Health Organization MONICA (monitoring trends and determinants in cardiovascular disease) project took place from the mid-1980s to mid-1990s. Across 38 populations in 21 countries in men and women aged 35-64, emphasising standardisation and quality control, the project related trends in mortality due to coronary disease, and in event rates, to trends in coronary care, and in risk factor levels in the population. Risk factor levels were measured through independent random sam- ple surveys in each population, at both ends of that decade, one of international concern with controlling hypertension. Most MONICA centres reported declining mean values in the populations for systolic and diastolic blood pressure; increases were exceptional. ${ }^{1-5}$

Epidemiologists identify two methods for changing continuous risk factors such as blood pressure. ${ }^{7}$ "High risk" targets the top of the population distribution bell curve, lowering blood pressure selectively in individuals where it is high. By contrast, through dietary, lifestyle, or environmental factors, "mass population" interventions (figure) deliberately or accidentally move the whole curve downwards, also lowering middle and low readings. Both change the population mean value. Can we apportion responsibility by pooling the 38 MONICA population results? Did the blood-pressure bell curve change position or shape? Did it swing, or was it dented? Did blood pressure fall or was it pushed?

\section{Methods}

\section{Blood pressure measurement}

Investigators followed standard protocols. ${ }^{38}$ In every population, with ethical approval, they targeted 200 or more participants from each 10 year age and sex group, from age 35 to 64 , for a random sample survey at the beginning of the MONICA decade, replicated independently at its end. ${ }^{2}$ Blood pressure was measured in the right arm by using a random zero or traditional mercury sphygmomanometer after seating the participant for five minutes. Systolic and diastolic blood pressures (phase 5) were recorded twice to the nearest $2 \mathrm{~mm} \mathrm{Hg}$ and the averages obtained. ${ }^{8}$ Data were forwarded to the MONICA data centre in Helsinki for analysis and quality assessment. ${ }^{38}$ The original database is available to MONICA investigators; detailed tabulations are in the public domain. ${ }^{5}$

\section{Statistical methods}

We extracted published information on numbers of participants and response rates, ${ }^{9}$ use of antihypertensive medication, ${ }^{5}$ scores assigned in the quality assessment of the blood pressure data, ${ }^{3}$ and summary statistics on numbers, blood pressure means, standard deviations, and previously defined low, middle, and high readings to be used for tracking change- the 20th, 50th and 80th centiles. ${ }^{5}$ Results for the age range 35-64 were standardised to the world standard population. ${ }^{140}$ We added selected analyses from the MONICA database.

Supplementary data, explanations for the tables, and a methodological appendix are on bmj.com 

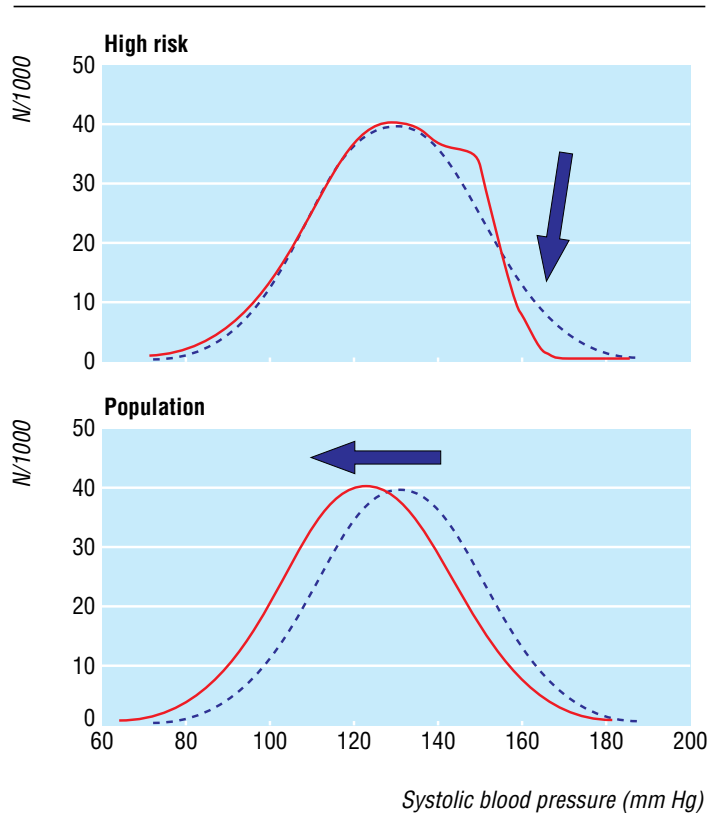

High risk interventions compared with mass population interventions: impact on an idealised population distribution or bell curve for systolic blood pressure

Subtraction of initial from final survey values means that a negative difference indicates a fall. We used differences obtained between surveys in individual populations to produce simple mean pooled values. The number of populations, generally 38 or 19 , was used to calculate the standard errors of these pooled means.

Our hypothesis was that hypertension control would selectively depress the top end of the population bell curve, resulting in pooled results showing differential falls in the 80th centile compared with the 20th centile; the mean compared with the 50th centile; and the mean compared with the average change in these three centiles, so we obtained these differences by subtraction. Null hypotheses of no treatment (but a mass population) effect would be disproved by significant values for these differences. In order to test the robustness of the results obtained from pooling all 38 populations together, we then compared the findings in contrasting subgroupings of these populations.

\section{Results}

Listings in table 1 (sexes combined) show by population and survey: numbers surveyed and the participation rates, ${ }^{9}$ use of antihypertensive medication, ${ }^{5}$ and also overall quality scores for blood pressure trends, ranging from 0.0 (potentially important problems) to 2.0 (no material problems detected). ${ }^{3}$ Replicate surveys averaged 8.9 (SD 1.2) years apart. ${ }^{11}$

Tables 2 (men) and 3 (women) show changes in systolic blood pressure and diastolic blood pressure. Results for the age range 35-64 are age standardised. Original tables and our derived spreadsheets are available (see appendix on bmj.com). ${ }^{5}$ Change in mean systolic and diastolic blood pressure in men averaged $-2.2 \mathrm{~mm} \mathrm{Hg}$ and $-1.4 \mathrm{~mm} \mathrm{Hg}$ (ranges -10.6 to $+4.5 \mathrm{~mm} \mathrm{Hg}$ and -9.5 to $+3.5 \mathrm{~mm} \mathrm{Hg}$ ); in women $-3.3 \mathrm{~mm} \mathrm{Hg}$ and -2.2 $\mathrm{mm} \mathrm{Hg}$, (ranges -13.5 to $+8.1 \mathrm{~mm} \mathrm{Hg}$ and -10.7 to $+3.9 \mathrm{~mm}$ $\mathrm{Hg}$ ). Changes in the mean and in different centiles vary considerably within and between populations. The overall drop in blood pressure after averaging falls in systolic and diastolic blood pressure and both sexes across populations was $-2.26 \mathrm{~mm} \mathrm{Hg}$, but the median population value was $-1.55 \mathrm{~mm} \mathrm{Hg}$ (see appendix on bmj.com). Despite the variation between populations, on taking all 38 populations together, the pooled average values of the changes in the mean and the changes in the centiles chosen for comparison, are remarkably similar.

Table 4 explores the null hypotheses. Changes in mean blood pressure are substantial and significant. Differences entailed in the null hypotheses are inconsistent between the sexes, between systolic blood pressure and diastolic blood pressure, and mostly approximate to zero, with non-significant positive or negative values. Older age groups are similar in results to age 35-44, where medication was rare.

Table 5 partitions the 38 populations into halves (usually 19:19), to show whether the null hypothesis differences remain non-significant when contrasting populations by level of antihypertensive medication, by change in medication, by size of blood pressure fall, by quality assessment score, by population sample size, and by national gross domestic product per head. ${ }^{12}$

The mean fall in blood pressure varies, but it differs significantly from zero in almost all subgroupings. By contrast, the differences used in testing the null hypotheses remain clustered around zero-the few exceptions are inconsistent between the three hypothesis tests and between systolic blood pressure and diastolic blood pressure. The most precise results, differences between the decline in the mean and centile average, do not imply a significant treatment effect contributing to the decline in mean blood pressure values.

If results from table 4 , systolic and diastolic blood pressure, both sexes, are averaged to give an overall value, then the mean change is $-2.26 \mathrm{~mm} \mathrm{Hg}$. The difference by subtraction between the change in the 20th and that in the 80th centile is $+0.01 \mathrm{~mm}$ $\mathrm{Hg}$, between the change in the 50th centile and the mean, +0.05 $\mathrm{mm} \mathrm{Hg}$; and between the change in the centile average and the mean, $+0.04 \mathrm{~mm} \mathrm{Hg}$.

Change in mean blood pressure is significantly negative. The null hypothesis differences are tending to be non-significantly positive where treatment benefit should have produced significant negative values. A contribution from improving control of hypertension to the overall population decline in blood pressure seems undetectable.

\section{Discussion}

The absence of a detectable contribution to the decline in blood pressure over the MONICA decade from improving control of hypertension was unexpected. Although this finding does not contradict trial evidence that antihypertensive medication limits cardiovascular risk in individuals, ${ }^{13}$ it implies that other factors, potentially of great public health interest, were more pervasive and powerful in lowering blood pressure across whole populations at that time. Welcome to those concerned with lifestyle or hygienic methods of controlling risk factors, these findings inevitably challenge interested parties who will want to ask searching technical questions. This short discussion is therefore supplemented by a methodological appendix of frequently asked questions (see bmj.com).

\section{Validity of analyses}

We are confident that our analyses are valid ${ }^{14}$ and alternatives, analysing those on and off medication separately are not. ${ }^{15}$ The WHO MONICA project is unique in the range and quality of its data, and in publishing detailed quality assessments along with its data books. ${ }^{1-5}$ There are no equivalent databases in which to replicate our analyses. However, by basing our main analyses on 
Research

Table 1 Populations, numbers in survey, response rates, percentage taking antihypertensive medication at each survey (age 35-64, age standardised, sexes combined), and quality assessment scores for each centre

\begin{tabular}{|c|c|c|c|c|c|c|c|c|}
\hline \multirow[b]{2}{*}{ Population } & \multicolumn{3}{|c|}{ Initial survey } & \multicolumn{3}{|c|}{ Final survey } & \multirow[b]{2}{*}{$\begin{array}{l}\text { Change in } \\
\text { participants taking } \\
\text { antihypertensive } \\
\text { medication (\%) }\end{array}$} & \multirow[b]{2}{*}{$\begin{array}{l}\text { Quality } \\
\text { assessmen } \\
\text { score }\end{array}$} \\
\hline & $\begin{array}{c}\text { No of } \\
\text { participants }\end{array}$ & $\begin{array}{l}\text { Response } \\
\text { rate (\%) }\end{array}$ & $\begin{array}{c}\% \text { of participants } \\
\text { taking } \\
\text { antihypertensive } \\
\text { medication }\end{array}$ & $\begin{array}{c}\text { No of } \\
\text { participants }\end{array}$ & $\begin{array}{l}\text { Response } \\
\text { rate (\%) }\end{array}$ & $\begin{array}{l}\% \text { of participants taking } \\
\text { antihypertensive } \\
\text { medication }\end{array}$ & & \\
\hline Australia-Newcastle & 2461 & 82 & 15.7 & 1325 & 77 & 14.3 & -1.4 & 1.0 \\
\hline Australia-Perth & 1292 & 83 & 11.2 & 609 & 82 & 9.4 & -1.8 & 0.0 \\
\hline Belgium-Charleroi & 815 & 43 & 10.2 & 867 & 44 & 9.8 & -0.4 & 2.0 \\
\hline Belgium-Ghent & 1028 & 57 & 11.3 & 1004 & 56 & 10.6 & -0.7 & 2.0 \\
\hline Canada-Halifax & 762 & 67 & 11.8 & 527 & 69 & 11.7 & -0.1 & 1.5 \\
\hline China-Beijing & 1247 & 90 & 8.5 & 1123 & 71 & 9.5 & 0.9 & 2.0 \\
\hline Czech Republic-Czech Republic & 1938 & 85 & 16.5 & 1840 & 78 & 15.2 & -1.3 & 0.0 \\
\hline Denmark-Glostrup & 2817 & 80 & 7.2 & 1218 & 74 & 7.1 & -0.1 & 1.0 \\
\hline Finland-Kuopio & 1967 & 85 & 13.6 & 1178 & 81 & 14.1 & 0.5 & 2.0 \\
\hline Finland-North Karelia & 2386 & 80 & 14.3 & 1103 & 78 & 13.5 & -0.8 & 2.0 \\
\hline Finland-Turku/Loimaa & 2487 & 85 & 10.5 & 1196 & 82 & 10.2 & -0.4 & 1.5 \\
\hline France-Lille & 1191 & 69 & 14.7 & 1149 & 76 & 15.2 & 0.5 & 0.0 \\
\hline France-Strasbourg & 1381 & 49 & 12.2 & 1079 & 51 & 14.1 & 1.9 & 1.5 \\
\hline France-Toulouse & 1323 & 70 & 9.7 & 1175 & 63 & 11.7 & 2.0 & 0.0 \\
\hline Germany-Augsburg rural & 1704 & 85 & 7.8 & 1691 & 83 & 11.3 & 3.6 & 2.0 \\
\hline German-Augsburg urban & 1388 & 80 & 7.9 & 1327 & 75 & 10.2 & 2.3 & 2.0 \\
\hline Germany-Bremen & 1291 & 71 & 7.2 & 826 & 66 & 12.7 & 5.5 & 2.0 \\
\hline Germany-East Germany & 816 & 90 & 15.7 & 921 & 59 & 14.2 & -1.5 & 1.5 \\
\hline Iceland-Iceland & 1341 & 76 & 9.0 & 1411 & 81 & 9.5 & 0.4 & 2.0 \\
\hline Italy-Brianza & 1269 & 71 & 10.2 & 1317 & 73 & 11.4 & 1.2 & 1.5 \\
\hline Italy-Friuli & 1459 & 84 & 10.6 & 1374 & 80 & 9.7 & -0.9 & 1.5 \\
\hline Lithuania-Kaunas & 1463 & 69 & 16.2 & 1239 & 76 & 10.4 & -5.9 & 2.0 \\
\hline New Zealand-Auckland & 1588 & 81 & 10.0 & 1472 & 74 & 8.0 & -2.1 & 2.0 \\
\hline Poland-Tarnobrzeg Voivodship & 2678 & 81 & 9.0 & 1317 & 76 & 13.4 & 4.5 & 0.0 \\
\hline Poland-Warsaw & 2624 & 74 & 10.7 & 1514 & 78 & 12.7 & 2.1 & 2.0 \\
\hline Russia-Moscow Control & 1416 & 78 & 13.1 & 1084 & 66 & 13.8 & 0.7 & 1.5 \\
\hline Russia-Moscow Intervention & 1175 & 70 & 15.3 & 1396 & 76 & 13.0 & -2.4 & 2.0 \\
\hline Russia-Novosibirsk Control & 1178 & 71 & 7.4 & 1184 & 70 & 20.7 & 13.4 & 1.0 \\
\hline Russia-Novosibirsk Intervention & 1267 & 73 & 12.9 & 1279 & 73 & 15.3 & 2.5 & 1.0 \\
\hline Spain-Catalonia & 1981 & 79 & 6.9 & 2609 & 76 & 8.8 & 1.9 & 2.0 \\
\hline Sweden-Gothenburg & 1039 & 74 & 7.9 & 1393 & 72 & 9.1 & 1.3 & 2.0 \\
\hline Sweden-Northern Sweden & 1260 & 86 & 10.1 & 1164 & 80 & 7.9 & -2.2 & 2.0 \\
\hline Switzerland-Ticino & 1550 & 83 & 8.0 & 1503 & 80 & 9.4 & 1.4 & 2.0 \\
\hline Switzerland-Vaud/Fribourg & 1197 & 69 & 7.6 & 1148 & 63 & 7.1 & -0.5 & 0.5 \\
\hline United Kingdom-Belfast & 1854 & 71 & 7.4 & 1609 & 50 & 6.4 & -1.1 & 2.0 \\
\hline United Kingdom-Glasgow & 984 & 65 & 7.0 & 1405 & 67 & 10.1 & 3.1 & 1.5 \\
\hline United States-Stanford & 958 & 69 & 10.9 & 1017 & 60 & 6.7 & -4.2 & 1.5 \\
\hline Yugoslavia-Novi Sad & 1182 & 82 & 16.8 & 1167 & 93 & 15.0 & -1.8 & 1.5 \\
\hline All & 1520 & 73 & 10.8 & 1257 & 71 & 11.4 & 0.5 & 1.5 \\
\hline
\end{tabular}

For specific survey dates see, reference 11; for derivation of quality assessment scores, see reference 3 ; for definition of response rate, see reference 9 .

published summary statistics ${ }^{5}$ and publishing our spreadsheets (see bmj.com) we have left a transparent audit trail by which others, if they wish, can rework them. We gave equal weight to all populations in pooling results. Our tests of robustness (table 5), imply that different weightings would produce similar answers.

Unfortunately for public health, we are unable to say what caused the blood pressure decline in MONICA. Other repeat surveys have found population blood pressures falling. Detailed discussion is beyond the scope of this paper, although doubtless claims will now be made for different nostrums. MONICA cannot give answers outside what it measured. Obesity, a known contributor, moved in the wrong direction. ${ }^{1}$ Possibilities, apart from measurement errors, ${ }^{3}{ }^{16}$ include cohort effects, ${ }^{17}$ dietary factors (including consumption of alcohol, sodium, potassium and other electrolytes), and other lifestyle and environmental factors. ${ }^{18}$

\section{Use of medication}

During the MONICA study, the use of antihypertensive medication in MONICA populations increased by a rounded average of $0.5 \%$, from $10.8 \%$ to $11.4 \%$ (see tables in bmj.com), but drugs in use changed. For $11.4 \%$ medication to be the exclusive cause of an average population-wide fall of $2.26 \mathrm{~mm} \mathrm{Hg}$ between surveys, the blood pressure fall of people taking medication would need to be an improbable $19.8 \mathrm{~mm} \mathrm{Hg}$. Individual data show that medication was concentrated among high readings: $25.5 \%$ usage above the 80 th centile and $3.4 \%$ below the 20 th.

Change in medication across populations and change in blood pressure were not correlated. In 21 market economy populations where blood pressure declined, use increased in 11 and decreased in 10, implying no simple association. Better blood pressure control in people taking medication could result from underlying population trends in blood pressure-the goal posts remaining stationary but the ground moving under them. 
Table 2 Differences in blood pressure in $\mathrm{mm} \mathrm{Hg}$ in men between the initial and final surveys: age 35-64 (age standardised)

\begin{tabular}{|c|c|c|c|c|c|c|c|c|c|c|c|c|}
\hline & \multicolumn{6}{|c|}{ Systolic blood pressure ${ }^{*}$} & \multicolumn{6}{|c|}{ Diastolic blood pressure* } \\
\hline & Mean & SD & $\begin{array}{c}\text { 20th } \\
\text { centile }\end{array}$ & $\begin{array}{c}\text { 50th } \\
\text { centile }\end{array}$ & $\begin{array}{c}\text { 80th } \\
\text { centile }\end{array}$ & $\begin{array}{l}\text { Centile } \\
\text { average }\end{array}$ & Mean & SD & $\begin{array}{c}\text { 20th } \\
\text { centile }\end{array}$ & 50th centile & $\begin{array}{c}\text { 80th } \\
\text { centile }\end{array}$ & $\begin{array}{r}\text { Centile } \\
\text { average }\end{array}$ \\
\hline Australia-Newcastle & -1.3 & -1.4 & -1.0 & 0.0 & -2.0 & -1.0 & -2.5 & -0.2 & -3.0 & -3.0 & -3.0 & -3.0 \\
\hline Australia-Perth & 0.0 & 1.1 & -2.0 & 1.0 & -1.0 & -0.7 & -0.1 & 1.1 & 0.0 & -1.0 & 1.0 & 0.0 \\
\hline Belgium-Charleroi & 0.5 & -0.9 & 0.0 & 1.0 & 2.0 & 1.0 & -0.8 & 0.1 & -2.0 & -1.0 & -1.0 & -1.3 \\
\hline Belgium-Ghent & 1.6 & 0.0 & 2.0 & 2.0 & 3.0 & 2.3 & 2.9 & 0.4 & 3.0 & 3.0 & 4.0 & 3.3 \\
\hline Canada-Halifax & 4.5 & 2.3 & 2.0 & 3.0 & 7.0 & 4.0 & 1.2 & -0.4 & 2.0 & 1.0 & 0.0 & 1.0 \\
\hline China-Beijing & 0.7 & -2.1 & 3.0 & 1.0 & 0.0 & 1.3 & -0.3 & -0.4 & 0.0 & 0.0 & -1.0 & -0.3 \\
\hline $\begin{array}{l}\text { Czech Republic-Czech } \\
\text { Republic }\end{array}$ & -0.6 & 1.5 & 0.0 & -3.0 & 0.5 & -0.8 & 0.5 & 0.5 & -1.0 & 2.0 & 1.0 & 0.7 \\
\hline Denmark-Glostrup & -1.1 & 0.5 & -1.0 & -3.0 & -1.0 & -1.7 & 3.0 & 0.4 & 2.0 & 3.0 & 3.0 & 2.7 \\
\hline Finland-Kuopio & -7.3 & -0.4 & -7.0 & -8.0 & -8.0 & -7.7 & -4.9 & -1.3 & -4.0 & -5.0 & -6.0 & -5.0 \\
\hline Finland-North Karelia & -2.1 & 0.9 & -3.0 & -4.0 & 0.0 & -2.3 & -1.9 & 0.0 & -2.0 & -3.0 & -4.0 & -3.0 \\
\hline Finland-Turku/Loimaa & -3.9 & -2.3 & -2.0 & -4.0 & -7.0 & -4.3 & -0.6 & -0.4 & 0.0 & -1.0 & -1.0 & -0.7 \\
\hline France-Lille & -4.5 & 0.1 & -4.0 & -6.0 & -5.0 & -5.0 & -5.6 & 0.2 & -4.0 & -6.0 & -6.0 & -5.3 \\
\hline France-Strasbourg & -9.5 & 0.4 & -9.0 & -9.0 & -10.0 & -9.3 & -4.5 & 1.4 & -6.0 & -4.0 & -4.0 & -4.7 \\
\hline France-Toulouse & -6.3 & -1.7 & -7.0 & -7.0 & -4.0 & -6.0 & -9.5 & -1.9 & -11.0 & -9.0 & -10.0 & -10.0 \\
\hline Germany-Augsburg rural & -0.1 & 0.4 & -1.0 & 1.0 & 0.0 & 0.0 & 0.5 & 0.0 & 0.0 & 0.0 & 1.0 & 0.3 \\
\hline German-Augsburg urban & 1.1 & 0.5 & 1.0 & 1.0 & 2.0 & 1.3 & -0.8 & -0.3 & -1.0 & -1.0 & -2.0 & -1.3 \\
\hline Germany-Bremen & -7.4 & -0.7 & -6.0 & -8.0 & -6.0 & -6.7 & -2.6 & -0.4 & -1.0 & -3.0 & -3.0 & -2.3 \\
\hline Germany-East Germany & 0.8 & 1.5 & -1.0 & 0.0 & 2.0 & 0.3 & 0.0 & 1.1 & -1.0 & -1.0 & 0.0 & -0.7 \\
\hline Iceland-Iceland & -0.8 & 0.0 & -2.0 & 1.0 & -1.0 & -0.7 & -1.0 & 1.5 & -4.0 & 0.0 & 0.0 & -1.3 \\
\hline Italy-Brianza & -6.1 & -0.1 & -5.0 & -6.0 & -5.0 & -5.3 & -3.4 & 1.1 & -4.0 & -4.0 & -3.0 & -3.7 \\
\hline Italy-Friuli & -2.0 & 0.2 & -2.0 & -3.0 & -3.0 & -2.7 & -0.8 & 0.0 & -1.0 & 0.0 & -1.0 & -0.7 \\
\hline Lithuania-Kaunas & 0.3 & -1.1 & 1.0 & 0.0 & -1.0 & 0.0 & 1.1 & 0.0 & 0.0 & 0.0 & 3.0 & 1.0 \\
\hline New Zealand-Auckland & -5.5 & -1.0 & -5.0 & -6.0 & -6.0 & -5.7 & -6.2 & -0.3 & -6.0 & -7.0 & -6.0 & -6.3 \\
\hline $\begin{array}{l}\text { Poland-Tarnobrzeg } \\
\text { Voivodship }\end{array}$ & -0.6 & -0.5 & -1.0 & 1.0 & -3.0 & -1.0 & -1.9 & 0.4 & -5.0 & -1.0 & -3.0 & -3.0 \\
\hline Poland-Warsaw & -10.6 & -0.3 & -11.0 & -10.0 & -11.0 & $\begin{array}{ll}-10.7 \\
\end{array}$ & -4.0 & 0.7 & -5.0 & -4.0 & -2.0 & -3.7 \\
\hline Russia-Moscow Control & -6.4 & -2.3 & -5.0 & -7.0 & -8.0 & -6.7 & -6.4 & -1.5 & -5.5 & -7.0 & -8.0 & -6.8 \\
\hline Russia-Moscow Intervention & -8.1 & -1.0 & -5.0 & -7.0 & -13.0 & -8.3 & -5.9 & -1.1 & -6.0 & -6.0 & -5.0 & -5.7 \\
\hline Russia-Novosibirsk Control & -0.4 & -1.2 & 1.0 & -4.0 & -1.0 & -1.3 & 0.3 & -1.0 & 2.0 & -2.0 & -2.0 & -0.7 \\
\hline $\begin{array}{l}\text { Russia-Novosibirsk } \\
\text { Intervention }\end{array}$ & 0.2 & 4.3 & -2.0 & -2.0 & 2.0 & -0.7 & -3.5 & -0.6 & -3.0 & -4.0 & -5.0 & -4.0 \\
\hline Spain-Catalonia & -2.5 & -1.2 & -1.0 & -2.0 & -4.0 & -2.3 & 0.0 & -1.1 & 1.0 & 0.0 & 0.0 & 0.3 \\
\hline Sweden-Gothenburg & 1.4 & 0.7 & 2.0 & 2.0 & 2.0 & 2.0 & 1.1 & 0.7 & 4.0 & 0.0 & 2.0 & 2.0 \\
\hline Sweden-Northern Sweden & -1.2 & 1.8 & -3.0 & -2.0 & 0.0 & -1.7 & 0.0 & 2.2 & -2.0 & -1.0 & 2.0 & -0.3 \\
\hline Switzerland-Ticino & -2.0 & -0.9 & -1.0 & -1.0 & -1.0 & -1.0 & 1.4 & -0.9 & 1.0 & 1.0 & 1.0 & 1.0 \\
\hline Switzerland-Vaud/ Fribourg & 0.1 & -1.0 & 2.0 & -1.0 & 0.0 & 0.3 & 1.8 & -0.5 & 2.0 & 2.0 & 1.0 & 1.7 \\
\hline United Kingdom-Belfast & 0.5 & -1.5 & 1.5 & 0.5 & 1.0 & 1.0 & 1.5 & -0.4 & 1.5 & 1.0 & 2.5 & 1.7 \\
\hline United Kingdom-Glasgow & -4.5 & -1.6 & -3.0 & -4.0 & -6.0 & -4.3 & -3.6 & -0.6 & -3.0 & -3.0 & -4.0 & -3.3 \\
\hline United States-Stanford & -0.8 & 0.5 & -1.5 & -0.5 & -1.5 & -1.2 & -0.5 & 0.9 & -1.0 & -0.5 & 0.5 & -0.3 \\
\hline Yugoslavia-Novi Sad & 1.0 & 4.1 & -2.0 & 0.5 & 5.5 & 1.3 & 3.5 & 3.0 & 1.0 & 4.5 & 4.0 & 3.2 \\
\hline Pooled mean & -2.2 & -0.1 & -2.1 & -2.4 & -2.1 & -2.2 & -1.4 & 0.1 & -1.6 & -1.6 & -1.4 & -1.5 \\
\hline $\begin{array}{l}\text { Lower } 95 \% \text { confidence limit } \\
\text { of pooled mean }\end{array}$ & -3.3 & -0.6 & -3.1 & -3.6 & -3.6 & -3.4 & -2.3 & -0.3 & -2.7 & -2.6 & -2.5 & -2.5 \\
\hline $\begin{array}{l}\text { Upper } 95 \% \text { confidence limit } \\
\text { of pooled mean }\end{array}$ & -1.1 & 0.4 & -1.0 & -1.3 & -0.7 & -1.1 & -0.4 & 0.4 & -0.6 & -0.6 & -0.3 & -0.6 \\
\hline
\end{tabular}

*Values are differences, derived by subtracting the initial survey statistic from that from the final survey. This explains, for example, why SD itself is not the standard deviation of the mean and has negative, and zero, as well as positive values.

This possibility is welcome, but potentially embarrassing for those publishing and interpreting analyses of repeated surveys studying the "rule of halves," the percentage of people with hypertension in the population identified, treated and controlled. ${ }^{19-21}$ In populations where blood pressure declined overall, with all else unchanged, the resulting improved percentages might be credited mistakenly to clinical vigilance and improved medication.

\section{Conclusions}

Our current findings, questioning the contribution of treatment, contrast with earlier MONICA collaborative results that showed unexpectedly strong links between population trends in medication for coronary care, and declining case fatality and mortality. ${ }^{22}$ This paradox results from our dispassionate analyses of standardised data across 38 different populations. But MONICA can report only on its own decade, now over. Medication may contribute more than spontaneous population change after the mid-1990s, although similar multicentre databases for analysis may not be available to show it. Data from the mid-1980s to mid1990s from the WHO MONICA project may still conceal surprises in other areas.

The MONICA population survey data book is available in the public domain from www.ktl.fi/publications/monica/surveydb/title.htm and in the CD Roms from the monograph; both contain lists of MONICA sites and key personnel, and significant sponsors. The survey data book originates with WHO MONICA Project investigators and thousands of participants.

Contributors. HTP conceived the study, organised and participated in the analyses, drafted and rewrote the manuscript, and is guarantor of the 
Table 3 Differences in blood pressure in $\mathrm{mm} \mathrm{Hg}$ in women between the initial and final surveys, age 35-64 (age standardised)

\begin{tabular}{|c|c|c|c|c|c|c|c|c|c|c|c|c|}
\hline & \multicolumn{6}{|c|}{ Systolic blood pressure* } & \multicolumn{6}{|c|}{ Diastolic blood pressure* $^{*}$} \\
\hline & Mean & SD & $\begin{array}{c}\text { 20th } \\
\text { centile }\end{array}$ & $\begin{array}{c}\text { 50th } \\
\text { centile }\end{array}$ & $\begin{array}{c}\text { 80th } \\
\text { centile }\end{array}$ & $\begin{array}{l}\text { Centile } \\
\text { average }\end{array}$ & Mean & SD & 20th centile & $\begin{array}{c}\text { 50th } \\
\text { centile }\end{array}$ & $\begin{array}{c}\text { 80th } \\
\text { centile }\end{array}$ & $\begin{array}{l}\text { Centile } \\
\text { average }\end{array}$ \\
\hline Australia-Newcastle & -1.7 & -1.5 & 1.0 & -2.0 & -5.0 & -2.0 & -3.4 & -1.5 & -2.0 & -4.0 & -5.0 & -3.7 \\
\hline Australia-Perth & -2.0 & 1.2 & -3.0 & -2.0 & -1.0 & -2.0 & -2.4 & 0.9 & -4.0 & -3.0 & -4.0 & -3.7 \\
\hline Belgium-Charleroi & -0.3 & 2.5 & -2.0 & -2.0 & 3.0 & -0.3 & -1.2 & 2.0 & -4.0 & -1.0 & 1.0 & -1.3 \\
\hline Belgium-Ghent & -0.3 & -2.3 & 2.0 & 2.0 & -1.0 & 1.0 & 1.4 & -0.5 & 2.0 & 3.0 & 1.0 & 2.0 \\
\hline Canada-Halifax & 8.1 & 0.8 & 8.0 & 6.0 & 10.0 & 8.0 & 3.9 & -1.2 & 5.0 & 4.0 & 3.0 & 4.0 \\
\hline China-Beijing & 1.5 & 1.7 & 0.0 & -1.0 & 5.0 & 1.3 & -0.9 & 1.5 & -3.0 & -1.0 & -1.0 & -1.7 \\
\hline $\begin{array}{l}\text { Czech Republic-Czech } \\
\text { Republic }\end{array}$ & -1.6 & 1.9 & -5.0 & -1.5 & 0.0 & -2.2 & -0.2 & 1.3 & -2.5 & -2.0 & 1.5 & -1.0 \\
\hline Denmark-Glostrup & -2.9 & -1.0 & -1.0 & -2.0 & -5.0 & -2.7 & 3.1 & 0.0 & 2.0 & 4.0 & 3.0 & 3.0 \\
\hline Finland-Kuopio & -6.6 & 0.1 & -6.0 & -6.0 & -9.0 & -7.0 & -4.1 & -0.3 & -4.0 & -4.0 & -4.0 & -4.0 \\
\hline Finland-North Karelia & -5.4 & 1.0 & -7.0 & -6.0 & -6.0 & -6.3 & -4.6 & -0.7 & -4.0 & -3.0 & -5.0 & -4.0 \\
\hline Finland-Turku/Loimaa & -2.9 & $\begin{array}{ll}-1.7 \\
\end{array}$ & 0.0 & -1.0 & -6.0 & -2.3 & 0.6 & -0.6 & 1.0 & 1.0 & 0.0 & 0.7 \\
\hline France-Lille & -4.5 & 0.1 & -3.0 & -4.5 & -6.0 & -4.5 & -5.4 & 0.5 & -9.0 & -5.0 & -5.0 & -6.3 \\
\hline France-Strasbourg & -10.6 & -0.6 & -10.0 & -10.0 & -10.0 & -10.0 & -6.2 & -0.1 & -7.0 & -7.0 & -6.0 & -6.7 \\
\hline France-Toulouse & -8.7 & -0.3 & -7.0 & -11.0 & -11.0 & -9.7 & -10.7 & -1.2 & -8.0 & -10.0 & -11.0 & -9.7 \\
\hline Germany-Augsburg rural & -3.1 & -0.8 & -4.0 & -2.0 & -2.0 & -2.7 & -1.0 & -0.2 & -1.0 & -1.0 & 0.0 & -0.7 \\
\hline German-Augsburg urban & 0.9 & 0.5 & 1.0 & -1.0 & 2.0 & 0.7 & -1.1 & -0.2 & -2.0 & -1.0 & 0.0 & -1.0 \\
\hline Germany-Bremen & -9.5 & -2.7 & -8.0 & -8.0 & -11.0 & -9.0 & -4.4 & -1.0 & -5.0 & -4.0 & -7.0 & -5.3 \\
\hline Germany-East Germany & -2.0 & 2.8 & -3.0 & -4.0 & 1.0 & -2.0 & 0.0 & 0.3 & 0.0 & -1.0 & 0.0 & -0.3 \\
\hline Iceland-Iceland & 1.3 & 0.7 & 0.0 & 0.0 & 4.0 & 1.3 & -0.7 & 1.2 & -2.0 & 0.0 & 0.0 & -0.7 \\
\hline Italy-Brianza & -5.9 & -0.7 & -5.0 & -4.0 & -7.0 & -5.3 & -4.1 & 1.3 & -5.0 & -3.0 & -1.0 & -3.0 \\
\hline Italy-Friuli & -3.3 & $\begin{array}{ll}-1.8 \\
\end{array}$ & -1.0 & -4.0 & -5.0 & -3.3 & -2.7 & -0.5 & -3.0 & -2.0 & -1.0 & -2.0 \\
\hline Lithuania-Kaunas & -3.0 & -1.0 & -3.0 & -2.0 & -4.0 & -3.0 & 0.3 & 0.2 & -1.0 & 1.0 & 0.0 & 0.0 \\
\hline New Zealand-Auckland & -3.0 & 1.4 & -5.0 & -3.0 & -2.0 & -3.3 & -5.6 & 0.7 & -6.0 & -5.0 & -5.0 & -5.3 \\
\hline $\begin{array}{l}\text { Poland-Tarnobrzeg } \\
\text { Voivodship }\end{array}$ & -3.0 & -0.1 & -5.0 & -1.0 & 1.0 & -1.7 & -5.0 & -0.5 & -7.0 & -5.0 & -7.0 & -6.3 \\
\hline Poland-Warsaw & -13.5 & -2.4 & -12.0 & -15.0 & -15.0 & -14.0 & -4.6 & -0.2 & -6.0 & -4.0 & -5.0 & -5.0 \\
\hline Russia-Moscow Control & -7.3 & -1.0 & -7.0 & -7.0 & -10.0 & -8.0 & -6.4 & -1.2 & -6.0 & -5.0 & -7.5 & -6.2 \\
\hline Russia-Moscow Intervention & -10.7 & -3.1 & -7.0 & -9.0 & -14.0 & -10.0 & -7.2 & -1.2 & -8.0 & -7.0 & -8.0 & -7.7 \\
\hline Russia-Novosibirsk Control & -1.8 & -3.2 & 1.0 & -4.0 & -1.0 & -1.3 & -0.8 & -1.6 & 0.0 & -1.0 & -6.0 & -2.3 \\
\hline $\begin{array}{l}\text { Russia-Novosibirsk } \\
\text { Intervention }\end{array}$ & 0.3 & 1.5 & 3.0 & 0.0 & 1.0 & 1.3 & -2.7 & -0.1 & -3.0 & -2.0 & -4.0 & -3.0 \\
\hline Spain-Catalonia & -3.6 & -1.3 & -3.0 & -3.0 & -4.0 & -3.3 & -0.6 & -0.6 & 0.0 & -1.0 & -1.0 & -0.7 \\
\hline Sweden-Gothenburg & 1.8 & 0.0 & 2.0 & 2.0 & 4.0 & 2.7 & 0.4 & 0.0 & 0.0 & 0.0 & 2.0 & 0.7 \\
\hline Sweden-Northern Sweden & -2.6 & 2.2 & -4.0 & -4.0 & 0.0 & -2.7 & -2.5 & -0.1 & -3.0 & -2.0 & -2.0 & -2.3 \\
\hline Switzerland-Ticino & -6.6 & -2.5 & -5.0 & -5.0 & -8.0 & -6.0 & $\begin{array}{ll}-1.6 \\
\end{array}$ & -0.7 & -1.0 & -1.0 & -2.0 & -1.3 \\
\hline Switzerland-Vaud/Fribourg & -2.6 & -2.0 & -1.0 & -2.0 & -3.0 & -2.0 & 0.6 & -0.5 & 1.0 & 1.0 & 0.0 & 0.7 \\
\hline United Kingdom-Belfast & -2.2 & -2.2 & 0.0 & -0.5 & -3.0 & -1.2 & 0.5 & -0.3 & 1.0 & 1.5 & 1.0 & 1.2 \\
\hline United Kingdom-Glasgow & -6.9 & -2.5 & -5.0 & -7.0 & -7.0 & -6.3 & -5.9 & -1.2 & -5.0 & -5.0 & -7.0 & -5.7 \\
\hline United States-Stanford & -3.6 & -0.1 & -4.5 & -3.5 & -4.5 & -4.2 & -1.2 & -0.1 & -1.5 & -1.0 & -0.5 & -1.0 \\
\hline Yugoslavia-Novi Sad & 2.3 & 4.9 & -2.0 & 1.5 & 5.0 & 1.5 & 3.8 & 1.9 & 1.5 & 4.0 & 4.0 & 3.2 \\
\hline Pooled mean & -3.3 & -0.3 & -2.9 & -3.3 & -3.3 & -3.2 & -2.2 & -0.1 & -2.6 & -1.9 & -2.3 & -2.3 \\
\hline $\begin{array}{l}\text { Lower } 95 \% \text { confidence limit } \\
\text { of pooled mean }\end{array}$ & -4.7 & -0.9 & -4.2 & -4.6 & -5.1 & -4.5 & -3.2 & -0.4 & -3.7 & -2.9 & -3.5 & -3.3 \\
\hline $\begin{array}{l}\text { Upper } 95 \% \text { confidence limit } \\
\text { of pooled mean }\end{array}$ & -2.0 & 0.3 & $\begin{array}{l}-1.7 \\
\end{array}$ & -2.1 & -1.5 & -1.8 & -1.1 & 0.2 & -1.6 & -0.9 & -1.2 & -1.2 \\
\hline
\end{tabular}

*Values listed are differences, derived by subtracting the initial survey statistic from that from the final survey. This explains, for example why SD is not the standard deviation of the mean and has negative, and zero, as well as positive values.

results. JC carried out the data extractions from the data book and the analyses of individual data, and contributed to the manuscript. MW carried out critical reviews of the statistical analyses and the manuscript. HT was responsible for the survey data book which was the basis of the study. KK is overall guarantor of the WHO MONICA project data, led the production of the data book, and criticised the study design and the manuscript. All authors share responsibility for the final drafts.

Funding: Work in Dundee, including that of John Connaghan, was supported from a British Heart Foundation programme grant.

Competing interests: None declared.

Ethical approval: Responsibility, and effected by principal investigators, in each population. This analysis is of anonymised data.

1 Tunstall-Pedoe $\mathrm{H}$, ed, for the WHO MONICA Project. MONICA monograph and multimedia sourcebook. Geneva: World Health Organization, 2003. www.ktl.fi/monica/ public/monograph.html (accessed 19 Feb 2006)

2 WHO MONICA Project. MONICA manual. 1998-1999. www.ktl.fi/publications/ monica/manual/index.htm (accessed 19 Feb 2006)
3 Kuulasmaa K, Hense H, Tolonen H, for the WHO MONICA Project. Quality assessment of data on blood pressure in the WHO MONICA Project. May 1998. www.ktl.fi/publications/ monica/bp/bpqa.htm (accessed 19 Feb 2006).

4 Kuulasmaa K, Tunstall-Pedoe H, Dobson A, Fortmann S, Sans S, Tolonen H, et al, for the WHO MONICA Project. Estimation of contribution of changes in classic risk factors to trends in coronary-event rates across the WHO MONICA project populations. Lancet 2000;355:675-87.

5 Tolonen H, Kuulasmaa K, Ruokokoski E, for the WHO MONICA Project. MONICA population survey data book. October 2000. www.ktl.fi/publications/monica/surveydb/ title.htm (accessed 19 Feb 2006).

6 Epstein FH. Coronary heart disease epidemiology revisited. Clinical and community aspects. Circulation 1973;48:185-9.

7 Rose G. Sick individuals and sick populations. Int J Epidemiol 1985;14:32-8.

8 WHO MONICA Project. MONICA manual. 1998-1999. Part III: population survey. Section 1: population survey data component. www.ktl.fi/publications/monica/manual/ part3/iii-1.htm (accessed 19 Feb 2006).

9 Wolf HK, Kuulasmaa K, Tolonen H, Ruokokoski E, for the WHO MONICA Project. Participation rates, quality of sampling frames and sampling fractions in the MONICA surveys. September 1998. www.ktl.fi/publications/monica/nonres/nonres.htm (accessed $19 \mathrm{Feb} 2006$ ). 
Table 4 Pooled blood pressure results from 38 populations: mean change between surveys, and the three differences used to test the null hypotheses, in $\mathrm{mm} \mathrm{Hg}$ (with $95 \%$ confidence intervals)

\begin{tabular}{|c|c|c|c|c|}
\hline All 38 populations & Mean & $\begin{array}{l}\text { 80th-20th } \\
\text { centile }\end{array}$ & $\begin{array}{l}\text { Mean-50th } \\
\text { centile }\end{array}$ & $\begin{array}{l}\text { Mean-centile } \\
\text { average }\end{array}$ \\
\hline \multicolumn{5}{|l|}{ Ages 35-44: } \\
\hline $\begin{array}{l}\text { Systolic blood } \\
\text { pressure }\end{array}$ & $\begin{array}{c}-2.72 \\
(-3.93 \text { to }-1.51)^{\star * *}\end{array}$ & $\begin{array}{c}-0.47 \\
(-1.14 \text { to } 0.20)\end{array}$ & $\begin{array}{c}0.03 \\
(-0.32 \text { to } 0.38)\end{array}$ & $\begin{array}{c}0.17 \\
(-0.09 \text { to } 0.42)\end{array}$ \\
\hline $\begin{array}{l}\text { Diastolic blood } \\
\text { pressure }\end{array}$ & $\begin{array}{c}-2.05 \\
(-2.97 \text { to }-1.13)^{\star \star \star *}\end{array}$ & $\begin{array}{c}0.05 \\
(-0.53 \text { to } 0.63)\end{array}$ & $\begin{array}{c}0.04 \\
(-0.22 \text { to } 0.30)\end{array}$ & $\begin{array}{c}0.12 \\
(-0.01 \text { to } 0.24)\end{array}$ \\
\hline \multicolumn{5}{|l|}{ Ages 45-54: } \\
\hline $\begin{array}{l}\text { Systolic blood } \\
\text { pressure }\end{array}$ & $\begin{array}{c}-2.74 \\
(-4.00 \text { to }-1.48)^{\star * *}\end{array}$ & $\begin{array}{c}-0.16 \\
(-1.13 \text { to } 0.81)\end{array}$ & $\begin{array}{c}0.37 \\
(0.01 \text { to } 0.73)^{*}\end{array}$ & $\begin{array}{c}0.01 \\
(-0.21 \text { to } 0.23)\end{array}$ \\
\hline $\begin{array}{l}\text { Diastolic blood } \\
\text { pressure }\end{array}$ & $\begin{array}{c}-1.63 \\
(-2.64 \text { to }-0.63)^{\star * *}\end{array}$ & $\begin{array}{c}0.18 \\
(-0.53 \text { to } 0.90)\end{array}$ & $\begin{array}{c}0.26 \\
(0.04 \text { to } 0.48)^{*}\end{array}$ & $\begin{array}{c}0.09 \\
(-0.06 \text { to } 0.24)\end{array}$ \\
\hline \multicolumn{5}{|l|}{ Ages 55-64: } \\
\hline $\begin{array}{l}\text { Systolic blood } \\
\text { pressure }\end{array}$ & $\begin{array}{c}-2.52 \\
(-4.04 \text { to }-1.01)^{\text {** }}\end{array}$ & $\begin{array}{c}0.36 \\
(-0.89 \text { to } 1.60)\end{array}$ & $\begin{array}{c}0.26 \\
(-0.16 \text { to } 0.68)\end{array}$ & $\begin{array}{c}-0.04 \\
(-0.22 \text { to } 0.14)\end{array}$ \\
\hline $\begin{array}{l}\text { Diastolic blood } \\
\text { pressure }\end{array}$ & $\begin{array}{c}-1.54 \\
(-2.68 \text { to }-0.41)^{\text {** }}\end{array}$ & $\begin{array}{c}-0.24 \\
(-0.94 \text { to } 0.47)\end{array}$ & $\begin{array}{c}0.12 \\
(-0.13 \text { to } 0.37)\end{array}$ & $\begin{array}{c}0.09 \\
(-0.03 \text { to } 0.20)\end{array}$ \\
\hline \multicolumn{5}{|l|}{ Men, age 35-64: } \\
\hline $\begin{array}{l}\text { Systolic blood } \\
\text { pressure }\end{array}$ & $\begin{array}{c}-2.18 \\
(-3.32 \text { to }-1.05)^{\star \star \star ~}\end{array}$ & $\begin{array}{c}-0.09 \\
(-1.00 \text { to } 0.81)\end{array}$ & $\begin{array}{c}0.25 \\
(-0.13 \text { to } 0.64)\end{array}$ & $\begin{array}{c}0.03 \\
(-0.13 \text { to } 0.19)\end{array}$ \\
\hline $\begin{array}{l}\text { Diastolic blood } \\
\text { pressure }\end{array}$ & $\begin{array}{c}-1.38 \\
(-2.34 \text { to }-0.42)^{\star *}\end{array}$ & $\begin{array}{c}0.21 \\
(-0.42 \text { to } 0.84)\end{array}$ & $\begin{array}{c}0.20 \\
(-0.04 \text { to } 0.44)\end{array}$ & $\begin{array}{c}0.16 \\
(0.02 \text { to } 0.30)^{*}\end{array}$ \\
\hline \multicolumn{5}{|l|}{$\begin{array}{l}\text { Women, age } \\
\text { 35-64: }\end{array}$} \\
\hline $\begin{array}{l}\text { Systolic blood } \\
\text { pressure }\end{array}$ & $\begin{array}{c}-3.30 \\
(-4.65 \text { to }-1.96)^{\star \star \star *}\end{array}$ & $\begin{array}{c}-0.37 \\
(-1.55 \text { to } 0.82)\end{array}$ & $\begin{array}{c}0.03 \\
(-0.42 \text { to } 0.47)\end{array}$ & $\begin{array}{c}-0.13 \\
(-0.33 \text { to } 0.06)\end{array}$ \\
\hline $\begin{array}{l}\text { Diastolic blood } \\
\text { pressure }\end{array}$ & $\begin{array}{c}-2.17 \\
(-3.20 \text { to }-1.14)^{\star \star \star *}\end{array}$ & $\begin{array}{c}0.29 \\
(-0.42 \text { to } 1.00)\end{array}$ & $\begin{array}{c}-0.29 \\
(-0.52 \text { to }-0.06)^{*}\end{array}$ & $\begin{array}{c}0.10 \\
(-0.10 \text { to } 0.30)\end{array}$ \\
\hline \multicolumn{5}{|l|}{$\begin{array}{l}\text { Both sexes, age } \\
\text { 35-64: }\end{array}$} \\
\hline $\begin{array}{l}\text { Systolic blood } \\
\text { pressure }\end{array}$ & $\begin{array}{c}-2.74 \\
(-3.95 \text { to }-1.53)^{\star \star \star *}\end{array}$ & $\begin{array}{c}-0.23 \\
(-1.14 \text { to } 0.68)\end{array}$ & $\begin{array}{c}0.14 \\
(-0.20 \text { to } 0.48)\end{array}$ & $\begin{array}{c}-0.05 \\
(-0.19 \text { to } 0.08)\end{array}$ \\
\hline $\begin{array}{l}\text { Diastolic blood } \\
\text { pressure }\end{array}$ & $\begin{array}{c}-1.78 \\
(-2.75 \text { to }-0.80)^{\star * *}\end{array}$ & $\begin{array}{c}0.25 \\
(-0.32 \text { to } 0.82)\end{array}$ & $\begin{array}{c}-0.05 \\
(-0.20 \text { to } 0.11)\end{array}$ & $\begin{array}{c}0.13 \\
(0.00 \text { to } 0.26)^{*}\end{array}$ \\
\hline
\end{tabular}

10 Waterhouse J, Muir CS, Correa P, Powell J, eds. Cancer incidence in five continents. Lyon: International Agency for Research on Cancer, 1976;3:456.

11 Kuulasmaa K, Tolonen H, Ferrario M, Ruokokoski E, for the WHO MONICA Project. Age, date of examination and survey periods in the MONICA surveys. May 1998. www.ktl.fi/publications/monica/age/ageqa.htm (accessed $19 \mathrm{Feb} 2006$ ).

12 The Conference Board and Groningen Growth and Development Centre. Total economy database. January 2006. www.ggdc.net/dseries/totecon.html (accessed 19th February 2006)

13 Guidelines Committee. 2003 European Society of Hypertension-European Society of Cardiology guidelines for the management of arterial hypertension. $J$ Hypertension 2003;21:1011-53

14 Egger M, Smith GD, Phillips AN. Meta-analysis: principles and procedures. BMJ 1997;315:1371-4

15 Bradford Hill A, Hill ID. Bradford Hill's principles of medical statistics. 12th ed. London: Edward Arnold, 1991:266-7.

16 Parati G, Mancia G. White coat effect: semantics, assessment and pathophysiological implications. J Hypertens 2003;21:545-53.

17 Barker DJP. Mothers, babies and disease in later life. London: BMJ Publishing Group, 1994 53.

18 Srinath Reddy K, Katan MN. Diet, nutrition and the prevention of hypertension and cardiovascular diseases. Public Health Nutrition 2004;7:167-86.

19 Wilber JA. The problem of undetected and untreated hypertension in the community. Bull NY Acad Med 1973;49:510-20.

20 Chen R, Tunstall-Pedoe H, Morrison C, Connaghan J, A'Brook R. Trends and social factors in blood pressure control in Scottish MONICA surveys 1986-95: the rule of halves revisited.J Hum Hypertension 2003;17:751-9.

21 Antikainen R, Moltchanov V, Chukwuma C, Kuulasmaa K, Marques-Vidal P, Sans S, et $\mathrm{al}$, for the WHO MONICA Project. Trends in the prevalence, awareness, treatment and control of hypertension. The WHO MONICA Project. Eur J Cardiovasc Prev Rehabil 2006;13:13-29.

22 Tunstall-Pedoe H, Vanuzzo D, Hobbs M, Mähönen M, Cepaitis Z, Kuulasmaa K, et al, for the WHO MONICA Project. Estimation of contribution of changes in coronary care to improving survival, event rates, and coronary heart disease mortality across the WHO MONICA Project populations. Lancet 2000;355:688-700.

(Accepted 24 December 2005)

doi $10.1136 /$ bmj.38753.779005.BE

Cardiovascular Epidemiology Unit, Institute of Cardiovascular Research, University of Dundee, Ninewells Hospital, Dundee DD1 9SY

Hugh Tunstall-Pedoe emeritus professor of cardiovascular epidemiology

John Connaghan research assistant
Table 5 Pooled blood pressure results from contrasted population groupings: mean change between surveys and the three differences used to test the null hypotheses, $\mathrm{mm} \mathrm{Hg}$ (with 95\% confidence intervals)

\begin{tabular}{|c|c|c|c|c|}
\hline $\begin{array}{l}\text { Contrasting } \\
\text { subgroups }\end{array}$ & Mean & 80th-20th centile & $\begin{array}{l}\text { Mean-50th } \\
\text { centile }\end{array}$ & $\begin{array}{l}\text { Mean-centile } \\
\text { average }\end{array}$ \\
\hline \multicolumn{5}{|l|}{ Most treated: } \\
\hline $\begin{array}{l}\text { Systolic blood } \\
\text { pressure }\end{array}$ & $\begin{array}{c}-3.95 \\
(-6.12 \text { to }-1.78)^{\star *}\end{array}$ & $\begin{array}{c}-0.08 \\
(-1.61 \text { to } 1.45)\end{array}$ & $\begin{array}{c}0.34 \\
(-0.24 \text { to } 0.92)\end{array}$ & $\begin{array}{c}0.05 \\
(-0.13 \text { to } 0.23)\end{array}$ \\
\hline $\begin{array}{l}\text { Diastolic blood } \\
\text { pressure }\end{array}$ & $\begin{array}{c}-2.99 \\
(-4.56 \text { to }-1.43)^{\star \star *}\end{array}$ & $\begin{array}{c}-0.09 \\
(-1.07 \text { to } 0.89)\end{array}$ & $\begin{array}{c}0.02 \\
(-0.21 \text { to } 0.25)\end{array}$ & $\begin{array}{c}0.28 \\
(0.08 \text { to } 0.47)^{\star \star *}\end{array}$ \\
\hline \multicolumn{5}{|l|}{ Least treated: } \\
\hline $\begin{array}{l}\text { Systolic blood } \\
\text { pressure }\end{array}$ & $\begin{array}{c}-1.54 \\
(-2.49 \text { to }-0.58)^{\star *}\end{array}$ & $\begin{array}{c}-0.38 \\
(-1.47 \text { to } 0.71)\end{array}$ & $\begin{array}{c}-0.06 \\
(-0.43 \text { to } 0.30)\end{array}$ & $\begin{array}{c}-0.16 \\
(-0.36 \text { to } 0.05)\end{array}$ \\
\hline $\begin{array}{l}\text { Diastolic blood } \\
\text { pressure }\end{array}$ & $\begin{array}{c}-0.56 \\
(-1.54 \text { to } 0.42)\end{array}$ & $\begin{array}{c}0.59 \\
(-0.03 \text { to } 1.22)\end{array}$ & $\begin{array}{c}-0.12 \\
(-0.34 \text { to } 0.10)\end{array}$ & $\begin{array}{c}-0.01 \\
(-0.17 \text { to } 0.14)\end{array}$ \\
\hline \multicolumn{5}{|c|}{ Increasing treatment: } \\
\hline $\begin{array}{l}\text { Systolic blood } \\
\text { pressure }\end{array}$ & $\begin{array}{c}-3.98 \\
(-5.89 \text { to }-2.08)^{\star \star \star *}\end{array}$ & $\begin{array}{c}-0.53 \\
(-1.31 \text { to } 0.26) \\
\end{array}$ & $\begin{array}{c}0.08 \\
(-0.45 \text { to } 0.62)\end{array}$ & $\begin{array}{c}-0.16 \\
(-0.34 \text { to } 0.03)\end{array}$ \\
\hline $\begin{array}{l}\text { Diastolic blood } \\
\text { pressure }\end{array}$ & $\begin{array}{c}-3.01 \\
(-4.33 \text { to }-1.70)^{\star \star \star}\end{array}$ & $\begin{array}{c}-0.13 \\
(-1.03 \text { to } 0.76)\end{array}$ & $\begin{array}{c}-0.07 \\
(-0.30 \text { to } 0.17)\end{array}$ & $\begin{array}{c}0.16 \\
(-0.06 \text { to } 0.38)\end{array}$ \\
\hline \multicolumn{5}{|c|}{ Decreasing treatment: } \\
\hline $\begin{array}{l}\text { Systolic blood } \\
\text { pressure }\end{array}$ & $\begin{array}{c}-1.50 \\
(-2.90 \text { to }-0.10)^{*}\end{array}$ & $\begin{array}{c}0.07 \\
(-1.63 \text { to } 1.76)\end{array}$ & $\begin{array}{c}0.20 \\
(-0.25 \text { to } 0.65)\end{array}$ & $\begin{array}{c}0.05 \\
(-0.14 \text { to } 0.25) \\
\end{array}$ \\
\hline $\begin{array}{l}\text { Diastolic blood } \\
\text { pressure }\end{array}$ & $\begin{array}{c}-0.54 \\
(-1.83 \text { to } 0.75)\end{array}$ & $\begin{array}{c}0.63 \\
(-0.10 \text { to } 1.36)\end{array}$ & $\begin{array}{c}-0.03 \\
(-0.25 \text { to } 0.19)\end{array}$ & $\begin{array}{c}0.10 \\
(-0.05 \text { to } 0.25)\end{array}$ \\
\hline \multicolumn{5}{|l|}{ Larger fall: } \\
\hline $\begin{array}{l}\text { Systolic blood } \\
\text { pressure }\end{array}$ & $\begin{array}{c}-5.48 \\
(-6.89 \text { to }-4.06)^{\star \star * *}\end{array}$ & $\begin{array}{c}-1.50 \\
(-2.71 \text { to }-0.29)^{*}\end{array}$ & $\begin{array}{c}-0.12 \\
(-0.56 \text { to } 0.32)\end{array}$ & $\begin{array}{c}-0.03 \\
(-0.23 \text { to } 0.17)\end{array}$ \\
\hline $\begin{array}{l}\text { Diastolic blood } \\
\text { pressure }\end{array}$ & $\begin{array}{c}-3.88 \\
(-5.08 \text { to }-2.68)^{\star \star \star ~}\end{array}$ & $\begin{array}{c}-0.03 \\
(-0.74 \text { to } 0.68)\end{array}$ & $\begin{array}{c}-0.14 \\
(-0.30 \text { to } 0.02)\end{array}$ & $\begin{array}{c}0.09 \\
(-0.08 \text { to } 0.26)\end{array}$ \\
\hline
\end{tabular}

Smaller fall:

\begin{tabular}{lcccc}
\hline $\begin{array}{l}\text { Systolic blood } \\
\text { pressure }\end{array}$ & -0.01 & 1.04 & 0.40 & -0.07 \\
\hline Diastolic blood & $(-0.91$ to 0.90$)$ & $(-0.12$ to 2.20$)$ & $(-0.11$ to 0.91$)$ & $(-0.27$ to 0.13$)$ \\
pressure & 0.32 & 0.53 & 0.04 & 0.18 \\
$(-0.46$ to 1.11$)$ & $(-0.40$ to 1.45$)$ & $(-0.23$ to 0.31$)$ & $(-0.02$ to 0.38$)$
\end{tabular}

Higher quality scorest:

\begin{tabular}{lllll}
\hline Systolic blood & -2.96 & -0.18 & -0.12 & -0.18
\end{tabular}

$\begin{array}{lllll}\text { pressure } & (-4.88 \text { to }-1.03)^{*} & (-1.45 \text { to } 1.09) & (-0.55 \text { to } 0.31) & (-0.40 \text { to } 0.04)\end{array}$

$\begin{array}{lcccc}\text { Diastolic blood } & -1.59 & 0.56 & -0.05 & 0.02 \\ \text { pressure } & (-2.80 \text { to }-0.39)^{*} & (-0.16 \text { to } 1.27) & (-0.23 \text { to } 0.13) & (-0.12 \text { to } 0.16)\end{array}$

pressure $\quad(-2.80$

\begin{tabular}{lllll}
\hline Systolic blood & -2.55 & -0.28 & 0.38 & 0.07
\end{tabular}

\begin{tabular}{llccc} 
pressure & $(-4.18 \text { to }-0.92)^{\star *}$ & $(-1.65$ to 1.10$)$ & $(-0.14$ to 0.89$)$ & $(-0.09$ to 0.23$)$ \\
\hline
\end{tabular}

$\begin{array}{lcccc}\begin{array}{l}\text { Diastolic blood } \\ \text { pressure }\end{array} & -1.94 & -0.03 & -0.04 & 0.23 \\ & (-3.53 \text { to }-0.36)^{*} & (-0.92 \text { to } 0.87) & (-0.30 \text { to } 0.21) & (0.02 \text { to } 0.44)^{*}\end{array}$

pressure $\quad(-3.53$

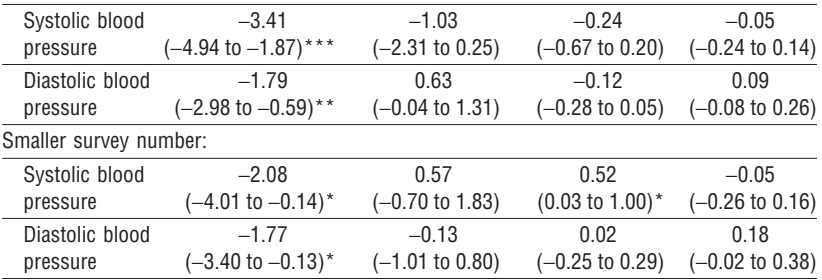

Larger gross domestic product†‡:

$\begin{array}{lllll}\text { Systolic blood } & -2.02 & 0.19 & 0.05 & -0.14\end{array}$

$\begin{array}{lllll}\text { pressure } \quad(-3.90 \text { to }-0.14)^{*} & (-0.85 \text { to } 1.24) & (-0.40 \text { to } 0.50) & (-0.35 \text { to } 0.08)\end{array}$

$\begin{array}{lllll}\text { Diastolic blood } & -1.34 & 0.40 & -0.03 & 0.08\end{array}$

\begin{tabular}{lllll} 
pressure & $(-2.91$ to 0.22$)$ & $(-0.35$ to 1.16$)$ & $(-0.25$ to 0.20$)$ & $(-0.07$ to 0.23$)$ \\
\hline
\end{tabular}

Smaller gross domestic product†:

\begin{tabular}{lllll}
\hline Systolic blood & -3.39 & -0.61 & 0.22 & 0.02
\end{tabular}

\begin{tabular}{lcccc} 
pressure & $(-5.01 \text { to }-1.77)^{\star \star *}$ & $(-2.11$ to 0.88$)$ & $(-0.30$ to 0.74$)$ & $(-0.15$ to 0.20$)$ \\
\hline
\end{tabular}

\begin{tabular}{lcccc}
$\begin{array}{l}\text { Diastolic blood } \\
\text { pressure }\end{array}$ & -2.17 & 0.11 & -0.07 & 0.18 \\
& $(-3.45 \text { to }-0.88)^{\star *}$ & $(-0.78$ to 1.00$)$ & $(-0.30$ to 0.16$)$ & $(-0.03$ to 0.39) \\
\hline
\end{tabular}

$\mathrm{P}=$ not significant $(>0.05)$ unless otherwise indicated; ${ }^{*} \mathrm{P} \leq 0.05$, ${ }^{*} \mathrm{P} \leq 0.01$, ${ }^{* *}{ }^{*} \mathrm{P} \leq 0.001$.

†18 $v 20$ populations. For other contrasting subgroups 38 populations are split 19:19.

$\ddagger$ Per head, for 1990, for the country concerned (reference 12).

George Institute for International Health, PO Box M201, Sydney, NSW 2050, Australia

Mark Woodward professor of biostatistics

International Cardiovascular Disease Epidemiology Unit, Department of Health Promotion and Chronic Disease Prevention, National Public Health Institute 


\section{What is already known on this topic}

Blood pressure is declining in many industrialised countries, but the mechanism is not known

The impact of treating hypertension on the population distribution of blood pressure is difficult to assess, as is the contribution to change over time

\section{What this study adds}

Populations differ in patterns of blood pressure decline

Pooled results from 38 populations show that antihypertensive medication made no detectable contribution to population decline in blood pressure in the mid-1980s to mid-1990s

Other determinants of blood pressure decline must have been more pervasive and powerful in the population as a whole during that decade

These findings are important in understanding blood pressure as a challenge to public health, but they do not deny the importance of antihypertensive medication in the individual
(KTL), 00300 Helsinki, Finland

Hanna Tolonen research fellow

Kari Kuulasmaa head of unit

Correspondence to: H Tunstall-Pedoe h.tunstallpedoe@dundee.ac.uk 